\title{
Organization Identifier
}

National Cancer Institute

\section{Source}

National Cancer Institute. Organization Identifier. NCI Thesaurus. Code C93401.

The unique identification of an organization in a specified context. 